\title{
El reto de la atención médica primaria previa y su asociación con la mortalidad por la COVID-19
}

\author{
The challenge of prior primary medical care and its \\ association with mortality from COVID-19
}

\author{
José Domingo Casillas-Enríquez,* Felipe de Jesús Cenobio-García,* Alejandro Efraín Benítez-Herrera ${ }^{\ddagger}$
}

*Subsecretaría de Servicios de Salud, Subdirección General de Prestación de Servicios de Hidalgo, México; ‡Secretaría de Salud del Estado de Hidalgo, México.

\begin{abstract}
RESUMEN. Introducción: La atención primaria conforma la puerta de entrada a los sistemas de salud. Durante la pandemia por la COVID-19, su labor es trascendental, ya que la identificación oportuna y la valoración adecuada de los casos impacta en su pronóstico y desenlace. Analizamos la atención previa a la hospitalización, como parte de la atención primaria. Identificamos un vacío en caracterizar la atención pública y privada, así como los tratamientos farmacológicos utilizados. Objetivo: Examinar la atención médica, pública y privada, el tipo de tratamiento farmacológico, previo al ingreso hospitalario y su asociación con la mortalidad por la COVID-19 en el estado de Hidalgo, México. Material y métodos: Se realizó un estudio multicéntrico, descriptivo y retrospectivo en pacientes hospitalizados en seis hospitales públicos del estado de Hidalgo, México. Se analizaron 596 casos, positivos a SARS-CoV-2. Se realizó la regresión logística binaria para examinar la relación entre la atención médica previa a la hospitalización y la mortalidad, así como para la relación entre el tipo de atención (pública y privada) y el tratamiento farmacológico. Resultados: $58 \%$ de los pacientes refirió atención previa a su ingreso hospitalario (86\% privada, 14\% pública). La mortalidad se relacionó con la edad, el sexo, la obesidad, insuficiencia renal crónica, atención previa y los días entre inicio de síntomas y la hospitalización. La atención médica privada se relacionó con el uso antibióticos ( $p<0.05)$. Conclusiones: La atención médica privada previa a la hospitalización por COVID-19 tiene un papel importante en la emergencia sanitaria. Es necesario capacitar al personal médico en estos sectores para propiciar la prescripción farmacológica adecuada, e implementar mecanismos de vigilancia epidemiológica de los casos sospechosos o descartados anticipadamente, como puntos críticos para mejorar la atención hospitalaria oportuna y disminuir la mortalidad.
\end{abstract}

Palabras clave: Atención primaria, mortalidad, COVID-19, México.

Correspondencia:

Dr. Felipe de Jesús Cenobio-García

Subsecretaría de Servicios de Salud,

Subdirección General de Prestación de Servicios.

Correo electrónico: felipe.cenobio@gmail.com

Recibido: 04-II-2021; aceptado: 20-V-2021.

Citar como: Casillas-Enríquez JD, Cenobio-García FJ, Benítez-Herrera AE. El reto de la atención médica primaria previa y su asociación con la mortalidad por la COVID-19. Neumol Cir Torax. 2021; 80 (2): 94-99. https://dx.doi. org/10.35366/100989
ABSTRACT. Introduction: Primary care is the gateway to health systems. During the COVID-19 pandemic, their work is transcendental since the timely identification and proper assessment of cases impacts their prognosis and outcome. We analyze care prior to hospitalization, as part of primary care. We identified a gap in characterizing public and private care, as well as the pharmacological treatments used. Objective: Examine public and private medical care, the type of pharmacological treatment, prior to hospital admission and its association with mortality from COVID-19 in the State of Hidalgo, Mexico. Material and methods: A multicenter, descriptive and retrospective study was carried out in hospitalized patients in six public hospitals in the State of Hidalgo, Mexico. 596 cases were analyzed, positive for SARS-CoV-2. Binary logistic regression was performed to examine the relationship between medical care prior to hospitalization and mortality, as well as the relationship between the type of care (public and private) and drug treatment. Results: $58 \%$ of the patients referred care prior to their hospital admission ( $86 \%$ private, $14 \%$ public). Mortality was related to age, sex, obesity, CKD chronic renal failure, previous care and the days between onset of symptoms and hospitalization. Private medical care was related to the use of antibiotics $(p<0.05)$. Conclusions: Private medical care prior to hospitalization for COVID-19 plays an important role in the health emergency. It is necessary to train medical personnel, in these sectors, to promote adequate pharmacological prescription, and implement epidemiological surveillance mechanisms for suspected or prematurely discarded cases, as critical points to improve timely hospital care and reduce mortality.

Keywords: Primary care, mortality, COVID-19, Mexico.

\section{INTRODUCCIÓN}

El síndrome respiratorio agudo severo coronavirus 2 (SARSCoV-2), la enfermedad resultante (COVID-19) y su rápida propagación mundial confirma hasta el 18 de octubre de 2020, 39 millones 596 mil 858 de casos y un millón 107 mil 374 defunciones. ${ }^{1}$

Al 11 de octubre de 2020, México se ubica entre los 10 primeros lugares en defunciones por COVID-19 en el mundo, con 648 defunciones por millón de habitantes. ${ }^{2}$ Se sabe que entre los factores de riesgo asociados con 
las complicaciones y la mortalidad por COVID-19, se encuentran la edad avanzada y las comorbilidades, ${ }^{3,4}$ ya que en el caso mexicano los pacientes fallecidos tenían una o múltiples comorbilidades, principalmente hipertensión $(45.5 \%)$, diabetes $(39.4 \%)$ y obesidad $(30 \%){ }^{5}$

Bajo este entendido, el contexto de población mórbida en Hidalgo, donde $12.8 \%$ padece diabetes y $18 \%$ hipertensión (población de 20 años y más) y 13.2\% obesidad (población de 12 a 19 años), ${ }^{6}$ la atención médica oportuna cobra importancia, por lo que se tiene el llamado constante de las autoridades de salud para que la población acuda a atención ante los primeros síntomas de la COVID-19. Sin embargo, los pacientes hospitalizados en el estado de Hidalgo, al corte del 19 de octubre de 2020, y que son positivos a SARS-CoV-2, acuden a atención médica hospitalaria, en promedio, seis días $(n=4,971)$ después del inicio de síntomas; 5.7 días para las defunciones $(n=2,033)$ y 6.1 días para los recuperados $(n=2,615)$, a pesar de que su media de edad es de 56 años y $71 \%$ de ellos presenta comorbilidades (29\% hipertensión, 28\% diabetes, 21\% obesidad y $4 \%$ insuficiencia renal crónica [IRC]). ${ }^{7}$

En este sentido, la atención primaria es trascendental, ya que conforma la puerta de entrada a los sistemas de salud. ${ }^{8,9}$ Esta situación no ha sido ajena durante la pandemia por la COVID-19, por lo que se ha resaltado que la atención sea oportuna, su importancia en la contención de los casos, ya que la identificación a tiempo y la valoración adecuada impacta en su pronóstico y desenlace. ${ }^{9-11}$ En este sentido, se ha mencionado la necesidad de fortalecer los servicios de atención primaria y el diseño y actualización de sus protocolos de respuesta, basados en evidencia disponible, para implementar estrategias prehospitalarias, por lo que se tienen lineamientos y flujogramas específicos. 8,12,13 También se han documentado las experiencias del manejo en atención primaria en pacientes con COVID-19. ${ }^{14}$ No obstante, es necesario diferenciar el tipo de atención y caracterizar la atención farmacológica previa a la hospitalización, como un componente de la atención primaria, para conocer su asociación con la mortalidad intrahospitalaria.

Se debe considerar que en 2015, 32\% de la población hidalguense, poco más de 861,600 personas no estaba afiliada a servicios médicos. Si bien $45 \%$ de ellos refirió que cuando tiene problemas de salud se atienden en centros de salud u hospitales de la Secretaría de Salud de Hidalgo, existe un sector importante (43\%) que opta por atenderse en consultorios o clínicas privadas; es decir, cerca de 373,600 personas. Por su parte, $1.9 \%$ refirió otro lugar y cerca de 29,100 personas que no se atiende. ${ }^{15}$ Esta situación, aunada al aumento de la atención médica en consultorios adyacentes a farmacias privadas y las condiciones precarias de los médicos que laboran en estos espacios (menor experiencia profesional, menos estudios de posgrado y menor salario promedio mensual), ${ }^{16}$ hace necesario examinar la atención médica, pública y privada, el tipo de tratamiento farmacológico previo al ingreso hospitalario y su asociación con la mortalidad por la COVID-19 en el estado de Hidalgo, México.

\section{MATERIAL Y MÉTODOS}

Se realizó un estudio multicéntrico, descriptivo y retrospectivo en pacientes hospitalizados entre el 29 de marzo y el 8 de octubre de 2020 en seis hospitales públicos, dependientes de la Secretaría de Salud del Estado de Hidalgo, México: Hospital General de Tula, Hospital General de Tulancingo, Hospital de Respuesta Inmediata Pachuca, Hospital General Pachuca, Hospital Regional del Valle del Mezquital Ixmiquilpan y Hospital de Respuesta Inmediata Actopan.

Se evaluaron 651 pacientes hospitalizados por sospecha de COVID-19 para su inclusión, de los cuales se incluyeron todos los positivos a SARS-CoV-2, por prueba de reacción en cadena de la polimerasa en tiempo real (RT-PCR). Se excluyeron aquellos con prueba negativa (48) y se eliminaron pacientes sin historia clínica o que estuviera incompleta o con ausencia de nota de ingreso (7), por lo que cumplieron criterios de inclusión 596 pacientes.

Como fuente de información se utilizó la base de datos clínica por sospecha de infección por SARS-CoV-2 de los hospitales de la Subsecretaría de Servicios de Salud y Subdirección General de Prestación de Servicios de la Secretaría de Salud del Estado de Hidalgo. Las variables se recopilaron de los expedientes clínicos (historia clínica, nota de ingreso, notas de evolución), los cuales se solicitaron a los hospitales de atención, y se recopilaron y guardaron en la base de datos, los médicos internos de pregrado las actualizaron diariamente. La recolección de datos se hizo bajo la supervisión de la Secretaría de Salud de Hidalgo. El respaldo, la confidencialidad y la seguridad de los datos personales se mantuvieron de acuerdo con las regulaciones mexicanas; los pacientes no estaban identificados antes del análisis. El análisis de datos y publicación de este estudio fue aprobado por la Secretaría de Salud de Hidalgo.

La base de datos se compone de las siguientes variables: hospital de atención, estado (defunción, alta por mejoría), edad, sexo, ocupación, estado y municipio de residencia, comorbilidades (enfermedad pulmonar obstructiva crónica [EPOC], diabetes mellitus; no se especifica el tipo, hipertensión, obesidad, cáncer, cardiopatías, IRC, insuficiencia hepática, enfermedad autoinmune, VIH), fecha de inicio de síntomas, fecha de hospitalización, fecha de egreso, resultado de prueba RT-PCR, atención previa (sí, no), lugar de la atención (público, privado), grupo farmacológico (nombre de los antiinflamatorios no esteroideos utilizados [AINE], nombre de los antibióticos, nombre de los antihistamínicos, nombre de los broncodilatadores, nombre de 
los mucolíticos y nombre de los corticoesteroides, todos éstos utilizados), automedicación (sí, no).

El análisis de las variables cualitativas se realizó calculando las frecuencias absolutas y relativas (en porcentaje). En el caso de las cuantitativas, se calculó la media y la desviación estándar.

Para examinar la relación entre la atención médica previa a la hospitalización y la mortalidad, así como para la relación entre el tipo de atención (pública y privada) y el tratamiento farmacológico, se realizó la regresión logística binaria. Para analizar el lapso, en días, entre el inicio de síntomas y el ingreso hospitalario, se realizó la prueba de

Tabla 1: Análisis descriptivo, frecuencias absolutas y porcentajes en pacientes positivos a SARS-CoV-2 hospitalizados en el estado de Hidalgo.

\begin{tabular}{|l|c|}
\hline Variable & $\mathrm{n}(\%)$ \\
\hline Sexo (N = 596) & \\
Hombres & $379(64)$ \\
Mujeres & $217(36)$ \\
\hline Edad, media \pm DE & $54 \pm 13.9$ \\
\hline Estado clínico (N = 596) & \\
Altas & $269(45)$ \\
Defunciones & $327(55)$ \\
\hline Estado de residencia (N =596) & \\
Hidalgo & $549(92)$ \\
Otro estado & $47(8)$ \\
\hline Comorbilidades (N=596) & \\
Sin comorbilidades & $136(23)$ \\
Una comorbilidad & $178(30)$ \\
Dos comorbilidades & $165(28)$ \\
Tres o más & $117(19)$ \\
\hline Obesidad & $282(47)$ \\
Diabetes & $258(43)$ \\
Hipertensión & $241(40)$ \\
EPOC & $34(6)$ \\
IRC & $30(5)$ \\
Cardiopatías & $25(4)$ \\
Enfermedad autoinmune & $8(1)$ \\
Enfermedad neurológica & $5(1)$ \\
Insuficiencia hepática & $2(0.3)$ \\
VIH & $1(0.2)$ \\
\hline Atención previa (N = 596) & $347(58)$ \\
Sí & $249(42)$ \\
No & \\
\hline Lugar de atención previa (N = 347) & \\
Privada & \\
Pública & \\
\hline
\end{tabular}

$\mathrm{EPOC}=$ enfermedad pulmonar obstructiva crónica; $\mathrm{IRC}=$ insuficiencia renal crónica; $\mathrm{VIH}=$ virus de la inmunodeficiencia humana.

Fuente: Elaboración propia. normalidad (Kolmogórov-Smirnov) y la prueba de diferencia de medias (Mann-Whitney). La significancia estadística elegida fue de $p<0.05$. Todos los análisis se realizaron en el software SPSS v.21.

\section{RESULTADOS}

De acuerdo con los criterios de exclusión y eliminación se incluyeron 596 pacientes para su análisis. Sus características se observan en la Tabla 1.

En cuanto a la atención farmacológica en ambos casos (pública y privada), 57\% fue tratado con AINE. En la atención prehospitalaria privada, $63 \%$ recibió tratamiento con antibióticos (de uno hasta cuatro, entre los que se encuentran: amikacina, amoxicilina, ampicilina, azitromicina, bencilpenicilina, cefaclor, cefalexina, cefixima, cefotaxima, ceftazidima, ceftriaxona, cefuroxima, ciprofloxacino, claritromicina, clindamicina, doxiciclina, eritromicina, gentamicina, imipenem, levofloxacino, lincomicina, moxifloxacino, trimetoprim/sulfametoxazol, oxitetraciclina), mientras que 49\% en los servicios públicos (de uno hasta tres antibióticos: amoxicilina, azitromicina, cefalexina, ceftriaxona, levofloxacino). El uso de antihistamínicos fue de 10\% en la privada, $4 \%$ en la pública. El $11 \%$ de los pacientes atendidos en el sector privado recibió tratamiento antiviral, respecto a $14 \%$ en la atención pública. En cuanto al uso de corticoesteroides, se administró en $9 \%$ en la atención privada y $8 \%$ en la pública (Tabla 2).

El promedio de días entre el inicio de síntomas y la hospitalización es de cinco para los que no tuvieron atención médica previa y ocho para los que sí. Los días entre el inicio de síntomas y la hospitalización no siguen una distribución normal $(p<0.05)$. A través de la prueba de Mann-Whitney se encontraron diferencias entre el inicio de síntomas y la hospitalización $(p<0.05)$.

Se realizó una regresión logística binaria para examinar los factores asociados con la mortalidad y otra para analizar el tipo de atención y el tratamiento farmacológico. En la primera, la mortalidad se relacionó con la edad, el sexo, la obesidad, IRC, la atención previa y los días entre inicio de síntomas y la hospitalización. En la segunda, la atención médica privada se asoció con el uso de antibióticos $(p<0.05)$ (Tabla 3).

\section{DISCUSIÓN}

Este trabajo muestra la importancia de la atención previa al ingreso hospitalario, con énfasis en los servicios privados de primer nivel. Existen características del contexto, como la creciente disponibilidad y uso de los servicios de salud en consultorios adyacentes a farmacias y características demográficas de la población analizada, como la ocupación, $55 \%$ de ésta engloba amas de casa, comerciantes, traba- 
Tabla 2: Análisis descriptivo del uso de medicamentos por grupo farmacológico, previo al ingreso hospitalario, positivos a SARS-CoV-2, Hidalgo.

\begin{tabular}{|l|c|c|c|}
\hline Grupo farmacológico & $\mathbf{n}(\%)$ & Mínimo & Máximo \\
\hline Atención privada (N = 298) & & & \\
Antiinflamatorios no esteroideos & $170(57)$ & 1 & 3 \\
Antibióticos & $189(63)$ & 1 & 4 \\
Antihistamínicos & $31(10)$ & 1 & 2 \\
Antivirales & $33(11)$ & 1 & 1 \\
Broncodilatadores & $31(10)$ & 1 & 2 \\
Mucolíticos & $39(13)$ & 1 & 1 \\
Corticoesteroides & $28(9)$ & 1 & 1 \\
\hline Atención pública (N =49) & & & \\
Antiinflamatorios no esteroideos & $28(57)$ & 1 & 3 \\
Antibióticos & $24(49)$ & 1 & 3 \\
Antihistamínicos & $2(4)$ & 1 & 1 \\
Antivirales & $7(14)$ & 1 & 2 \\
Broncodilatadores & $3(6)$ & 1 & 1 \\
Mucolíticos & $3(6)$ & 1 & 1 \\
Corticoesteroides & $4(8)$ & 1 & 1 \\
\hline
\end{tabular}

Fuente: Elaboración propia.

jadores agrícolas y empleados en establecimientos, lo cual nos hace pensar que la mayor parte de los casos recurrió a estos servicios. No obstante, es necesario profundizar para contrastar esta hipótesis en estudios posteriores.

Si bien la atención primaria pública y privada muestran similitudes en los tipos de tratamiento, encontramos una relación, estadísticamente significativa, entre el uso de antibióticos y la atención privada. El uso indiscriminado de antibióticos, con una amplia gama en este sector, no está indicado de primera línea en una afección viral, además de que su uso indiscriminado genera resistencia y neutraliza sus efectos terapéuticos, siendo necesarios tratamientos más complejos y prolongados en caso de una sobreinfección bacteriana. Lo anterior pone de manifiesto la necesidad de la capacitación adecuada del personal médico de primer contacto, como uno de los puntos críticos para mejorar la atención hospitalaria oportuna. Se tienen limitaciones al evaluar el uso de corticoesteroides (9\% en atención privada y $8 \%$ en la pública), dado que no contamos con información de la saturación de oxígeno al momento de la prescripción, no tenemos elementos para evaluar si se indicó por presentar hipoxemia, de no ser así, su uso puede contribuir a enmascarar la sintomatología de la COVID-19 y retrasar la atención médica hospitalaria oportuna.

Existen factores asociados con la mortalidad por COVID-19 que han sido ampliamente estudiados como son: edad, sexo y comorbilidades (diabetes, obesidad, hipertensión, IRC, por mencionar algunas)..$^{3-5,17,18}$ En concordancia, el modelo de regresión logística binaria encontró significancia en las siguientes variables: edad, sexo, obesidad, IRC, atención previa, días entre inicio de síntomas y la hospitalización. No se encontró significancia en la hipertensión y la diabetes, por lo que es necesario mencionar que los resultados deben interpretarse de manera prudente, ante la posibilidad de falta de poder estadístico en los casos analizados.

En este sentido, es importante mencionar algunas limitantes en el estudio, primero: el diseño retrospectivo implica un riesgo de sesgo en la información, en la memoria del paciente entrevistado y poder especificar el tratamiento asignado, segundo: los datos recabados dependen del adecuado llenado del expediente clínico, y de lo que el paciente refiere en la historia clínica.

Otro hallazgo relevante es la relación entre los días transcurridos entre el inicio de síntomas, el ingreso hospitalario y la mortalidad por la COVID-19. En este sentido, los estudios sobre la atención primaria han documentado la importancia de la valoración médica adecuada considerando el riesgo de complicación y el ingreso hospitalario oportuno. ${ }^{9-11}$ En este estudio, se observa una diferencia de tres días, en promedio, entre el inicio de síntomas y el ingreso hospitalario entre aquellos pacientes que tuvieron atención previa y los que no. Sin embargo, se desconocen los motivos del ingreso tardío, lo que puede ser consecuencia del enmascaramiento de síntomas, la falta de seguimiento y monitoreo de sín-

Tabla 3: Regresión logística binaria de factores asociados con la mortalidad por COVID-19, y tipos de atención y tratamiento farmacológico.

\begin{tabular}{|l|c|c|c|}
\hline Variable & OR & IC 95\% & p \\
\hline \multicolumn{3}{|l|}{} \\
\hline Mortalidad (alta por mejoría = 0; defunción = 1) \\
Edad & 1.06 & $1.04-1.07$ & $<0.05$ \\
Sexo (hombre) & 1.53 & $1.05-2.22$ & $<0.05$ \\
EPOC & 1.10 & $0.48-2.51$ & $>0.05$ \\
Diabetes & 0.95 & $0.65-1.37$ & $>0.05$ \\
Hipertensión & 0.85 & $0.57-1.26$ & $>0.05$ \\
Obesidad & 1.58 & $1.10-2.29$ & $<0.05$ \\
IRC & 3.10 & $1.15-8.39$ & $<0.05$ \\
Atención previa & 2.46 & $1.67-3.61$ & $<0.05$ \\
Días entre inicio de síntomas & 0.94 & $0.91-0.98$ & $<0.05$ \\
y hospitalización & \multicolumn{3}{|c|}{} \\
\hline Tipos de atención (pública =0; privada =1) y tratamiento farmacológico \\
\hline AINEs & 0.55 & $0.25-1.21$ & $>0.05$ \\
Antibióticos & 2.35 & $1.06-5.25$ & $<0.05$ \\
Antihistamínicos & 2.33 & $0.52-10.52$ & $>0.05$ \\
Antivirales & 0.55 & $0.21-1.43$ & $>0.05$ \\
Corticoesteroides & 1.08 & $0.34-3.39$ & $>0.05$ \\
Broncodilatadores & 1.62 & $0.46-5.72$ & $>0.05$ \\
Mucolíticos & 1.96 & $0.55-6.95$ & $>0.05$ \\
\hline
\end{tabular}

$E P O C$ = enfermedad pulmonar obstructiva crónica; IRC = insuficiencia renal crónica; AINEs = medicamentos antiinflamatorios no esteroideo.

Fuente: Elaboración propia. 
tomas, o la idiosincrasia de las personas que recibieron la atención primaria.

Se han documentado estrategias para optimizar la atención domiciliaria en pacientes con COVID-19 e identificar a aquellos que requieren atención médica hospitalaria de manera oportuna. ${ }^{14}$ Pero la atención médica privada carece de mecanismos de vigilancia epidemiológica para el seguimiento de casos sospechosos o que han sido descartados anticipadamente a COVID-19, por la valoración médica de primer contacto. También es imprescindible documentar si la atención primaria privada es un fenómeno característico en el estado de Hidalgo, aunque la evidencia señala que en el caso de la atención en consultorios adyacentes a farmacias es un fenómeno creciente en México. ${ }^{16}$ Por lo tanto, es una línea de investigación a retomar en otros estados del país, o en países donde este fenómeno se presente.

\section{CONCLUSIONES}

La atención médica previa al ingreso hospitalario juega un papel crucial en el tratamiento y pronóstico de los pacientes con COVID-19. Uno de los aportes es enfatizar la importancia de la capacitación adecuada del personal médico de primer contacto, con énfasis en la atención privada, ya que en nuestros hallazgos evidenciamos el uso excesivo de antibióticos ante una enfermedad de tipo viral, con un OR de 2.4 en la atención médica privada versus la atención médica pública. En este sentido, uno de los puntos críticos a mejorar es la atención primaria con apego a la evidencia científica y las guías clínicas de manejo avaladas a nivel internacional y nacional. Además, es necesario implementar mecanismos que propicien la adecuada vigilancia epidemiológica y seguimiento de los casos sospechosos a COVID-19, valorados de primer contacto en la medicina privada, lo que contribuirá a la derivación y hospitalización oportuna, impactando en la morbimortalidad de los pacientes.

\section{Agradecimientos}

A la Secretaría de Salud del Estado de Hidalgo.

\section{REFERENCIAS}

1. Secretaría de Salud. Informe técnico diario COVID-19 México [Internet]. México: Secretaría de Salud; 18/10/2020 [Consultado 19 octubre 2020]. Disponible en: https://www.gob.mx/cms/ uploads/attachment/file/585938/Comunicado_Tecnico_Diario_ COVID-19 2020.10.18.pdf

2. Secretaría de Salud. Conferencia de prensa, informe diario sobre coronavirus COVID 19 en México [Internet]. México: Secretaría de Salud; 13/10/2020 [Consultado 19 octubre 2020]. Disponible en: https://www.youtube.com/watch?v=ULMOpoHe-8s
3. Giannouchos TV, Sussman RA, Mier JM, Poulas K, Farsalinos K. Characteristics and risk factors for COVID-19 diagnosis and adverse outcomes in Mexico: an analysis of 89,756 laboratory-confirmed COVID-19 cases. Eur Respir J [internet]. 2020 [Consultado 10 de agosto de 2020]. Disponible en: https://doi.org/10.1183/13993003.021442020.

4. Simonnet A, Chetboun M, Poissy J, Raverdy V, Noulette J, Duhamel $A$, et al. High prevalence of obesity in severe acute respiratory syndrome coronavirus-2 (SARS-CoV-2) requiring invasive mechanical ventilation. Obesidad (Silver Spring) [internet]. 2020 [Consultado 10 de agosto de 2020];28(10):1994. Disponible en: https://doi.org/10.1002/ oby. 22831

5. Suárez V, Suárez Quezada M, Oros Ruiz S, Ronquillo De Jesús E. Epidemiología de COVID-19 en México: del 27 de febrero al 30 de abril de 2020. Rev Clin Esp [Internet]. 2020 [Consultado 25 de julio de 2020]; 220 (8): 463-471. doi: 10.1016/j.rce.2020.05.007.

6. Instituto Nacional de Salud Pública, Instituto Nacional de Estadística y Geografía. Encuesta Nacional de Salud y Nutrición. México: 2018. Disponible en: https://ensanut.insp.mx/encuestas/ ensanut2018/doctos/informes/ensanut_2018_presentacion_ resultados.pdf

7. Secretaría de Salud de Hidalgo. Listado Nominal de casos positivos a COVID-19, corte 19 de octubre 2020.

8. Sánchez-Duque JA, Arce-Villalobos LR, Rodríguez-Morales AJ. Enfermedad por coronavirus 2019 (COVID-19) en América Latina: papel de la atención primaria en la preparación y respuesta. Aten Primaria. 2020;52(6):369-372. Disponible en: http://dx.doi. org/10.1016/j.aprim.2020.04.001

9. Krist AH, Devoe JE, Cheng A, Ehrlich T, Jones SM. Rediseño de la atención primaria para abordar la pandemia COVID-19 en medio de la pandemia. Ann Fam Med. 2020;18(4):349-354. Disponible en: https://doi.org/10.1370/afm.2557

10. Sosa GJO. Atención de pacientes con COVID-19 en el consultorio médico. Rev CONAMED. 2020;25(S1):4-14. Disponible en: https:// doi.org/10.35366/97343

11. Llor C, Moragas A. Coronavirus y atención primaria. Aten Primaria. 2020;52(5):294-296. Disponible en: https://doi.org/10.1016/j. aprim.2020.03.002

12. Sarti T, Lazarini W, Fontenelle L, Almeida A. Qual o papel da atencao primária a saúde diante da pandemia provocada pela COVID-19? Epidemiol Serv Saúde [Internet]. 2020 [consultado 17 de marzo de 2021]. Disponible en: http://dx.doi.org/10.5123/s167949742020000200024

13. Haldane V, Zhang Z, Abbas RF, Dodd W, Lau LL, Kidd MR, et al. National primary care responses to COVID-19: a rapid review of the literature. BMJ Open. 2020;10(12):e041622. Available in: https://doi. org/10.1136/bmjopen-2020-041622

14. Blazey-Martin D, Barnhart E, Gillis J Jr, Vazquez GA. primary care population management for COVID-19 patients. J Gen Intern Med. 2020;35(10):3077-3080. Available in: https://doi.org/10.1007/s11606020-05981-1

15. Instituto Nacional de Estadística y Geografía. México: Microdatos de la Encuesta Intercensal; 2015.

16. Díaz-Portillo SP, Idrovo AJ, Dreser A, Bonilla FR, Matías-Juan B, Wirtz Veronika J. Consultorios adyacentes a farmacias privadas en México: infraestructura y características del personal médico y su remuneración. Salud Pública Méx. 2015;57(4):320-328. Disponible en: https://doi.org/10.21149/spm.v57i4.7575 
17. Chen T, Wu D, Chen H, Yan W, Yang D, Chen G, et al. Clinical characteristics of 113 deceased patients with coronavirus disease 2019: retrospective study. BMJ [Internet]. 2020 [Consultado 17 de septiembre de 2020];368:m1091. Disponible en: https://dx.doi. org/10.1136/bmj.m1091

18. Ruan R, Yang K, Wang W, Jiang L, Song J. Clinical predictors of mortality due to COVID-19 based on an analysis of data of 150 patients from Wuhan, China. Intensive Care Med [Internet]. 2020 [Consultado 20 de agosto de 2020]. Disponible en: https://link.springer.com/ article/10.1007\%2Fs00134-020-05991-x

Conflicto de intereses: Los autores declaran no tener conflicto de intereses. 